\title{
RADIO RECOMBINATION LINES FROM STARBURST GALAXIES
}

\author{
K.R. ANANTHARAMAIAH \\ Raman Research Institute, Bangalore 560 080, India
}

Starburst activity is often heavily obscured by dust. To see through the dust and measure the full extent, power and dynamics of the starburst, we are surveying hydrogen radio recombination lines (RRLs) from famous nearby starburst galaxies. Exploiting the improved sensitivity and dynamic range of aperture synthesis arrays such as the VLA and the AT and millimeter-wave telescopes such as SEST and the IRAM-30m telescope, we have searched for RRLs in about 25 starburst galaxies. RRLs have been detected in 14 galaxies. The observations were made variously at 1.4, 4.9, 8.4, 86, 135 and $232 \mathrm{GHz}$ (Anantharamaiah et al 1993, Zhao et al 1996, 1997 , Phookun et al 1997). Table 1 summarizes the observations. In all the cases the detected line originates in the nuclear starburst region and the emission region is resolved with a beam of $1-3^{\prime \prime}$.

TABLE 1. Summary of Recombination line Observations

\begin{tabular}{llc}
\hline Telescope & Galaxies Observed (Detections are in bold) & $\nu(G H z)$ \\
\hline VLA & NGC 253, NGC 262, NGC 520, NGC 660, & \\
& NGC 1068, NGC 1365, NGC 1614, NGC 2146, & \\
& NGC 3079, NGC 3628, NGC 3690, NGC 6240, & $8-9$ \\
& NGC 6946, NGC 7582, M 83, Arp 220, & \\
& IC 694 & \\
ATCA & NGC 1808, NGC 1068, NGC 3256, NGC 4945, & $8-9$ \\
& NGC 6221, NGC 7552, Circinus & $90,135,230$ \\
SEST & NGC 253, NGC 4945 NGC 1365, Circinus & 145 and 230 \\
\hline
\end{tabular}

The line and continuum strengths from the starburst regions are used to constrain the physical conditions such as temperature, density and mass 
of the ionized gas. In order to be consistent with the predominantly nonthermal radio spectrum of the nuclear region, the thermal gas that gives rise to the observed recombination line has to arise in a collection of high density $\left(>10^{3} \mathrm{~cm}^{-3}\right)$ compact HII regions. The HII regions occupy only a small fraction of the volume in the nuclear region with filling factors $\sim 10^{-4}-10^{-6}$. The derived parameters provide information about the number of high-mass stars formed in the starburst and the flux of Lyman continuum photons. Table 2 gives the observed and derived parameters for some of the galaxies.

TABLE 2. Some Observed and Derived Parameters (for $T_{e}=5000 \mathrm{~K}$ )

\begin{tabular}{lccccccc}
\hline Galaxy & Dist & $\begin{array}{c}\text { H92 } \alpha \text { Flux } \\
10^{-23}\end{array}$ & $\begin{array}{c}\text { Size } \\
\mathrm{W} \mathrm{m}^{-2}\end{array}$ & $\begin{array}{c}\text { Density } \\
10^{3}\end{array}$ & $\begin{array}{c}\text { Filling } \\
\text { factor } \\
\mathrm{cm}^{-3}\end{array}$ & $\begin{array}{c}M_{H I I} \\
10^{5}\end{array}$ & $\begin{array}{c}N_{L y c} \\
10^{53} \\
\mathrm{~s}_{\odot}\end{array}$ \\
\hline NGC 3628 & 11.5 & 8.6 & 500 & 50 & $3 \times 10^{-7}$ & 0.34 & 9.2 \\
IC 694 & 40 & 3.9 & 950 & 5 & $1 \times 10^{-5}$ & 5.7 & 16 \\
NGC 1365 & 22 & 12.0 & 1500 & 5 & $1 \times 10^{-6}$ & 2.3 & 6.2 \\
Arp 220 & 74 & 3.5 & 1900 & 10 & $3 \times 10^{-7}$ & 2.0 & 12 \\
M83 & 6.7 & 2.8 & 530 & 10 & $3 \times 10^{-5}$ & 0.06 & 0.33 \\
NGC 2146 & 12.8 & 2.7 & 370 & 10 & $2 \times 10^{-6}$ & 0.1 & 0.68 \\
NGC 253 & 3.4 & 44.5 & 90 & 10 & $2 \times 10^{-2}$ & 2.2 & 1.2 \\
NGC 660 & 11.3 & 5.6 & 640 & 5 & $4 \times 10^{-6}$ & 0.67 & 1.8 \\
\hline
\end{tabular}

The angular resolution of some of the recombination line observations are in the range $1^{\prime \prime}-3^{\prime \prime}$ which allows the study of kinematics in the nuclear region and an estimation of the dynamical mass. The estimated dynamical masses are generally $10^{3}-10^{4}$ times the ionized gas mass. In almost all cases in which the line region is resolved, the observed kinematics in the nuclear region indicate a rotating cirumnuclear disk. In a few cases, anomolous velocity features are observed. In the case of NGC 253 , the observed two dimensional velocity field can be interpreted in terms of two perpendicular rotating rings and a counter-rotating ring of ionized gas within the central $100 \mathrm{pc}$. It could also be interpreted in terms of a secondary bar inside the known primary bar (Anantharamaiah and Goss 1996).

\section{References}

Anantharamaiah, K.R., Zhao, J.H., Goss, W.M. and Viallefond, F. 1993, Ap.J., 419, 585 Anantharamaiah, K.R. and Goss, W.M. 1996, Ap.J., 466, L13

Phookun, B., Anantharamaiah, K.R. and Goss, W.M. 1997, MNRAS (in press)

Zhao, J.H., Anantharamaiah, K.R., Goss, W.M. and Viallefond, F. 1996, Ap.J., 472, 54

Zhao, J.H., Anantharamaiah, K.R., Goss, W.M. and Viallefond, F. 1997, Ap.J., 482, 186 